\title{
Effects of the Essential Oils of Sweet Orange, Lavender and Amyris on EEG Activity
}

\author{
Yerin $\mathrm{Je}^{1,2}$, Sungkwan $\mathrm{An}^{1}$, Hyangseon $\mathrm{Ro}^{2}$, Jawun $\mathrm{Cho}^{2}$, Seunghee $\mathrm{Bae}^{\mathrm{1}^{*}}$ \\ ${ }^{1}$ Department of Cosmetics Engineering, Konkuk University, Seoul, Korea \\ ${ }^{2}$ Research Center, Nowcos co., Ltd., Seoul, Korea
}

\author{
"Corresponding author: Seunghee Bae, \\ Department of Cosmetics Engineering, \\ Konkuk University, 120 Neungdong-ro, \\ Gwangjin-gu, Seoul 05029, Korea \\ Tel.: +82 24500463 \\ Email: sbae@konkuk.ac.kr
}

Received October 05, 2021

Revised October 22, 2021

Accepted November 17, 2021

Published December 30, 2021

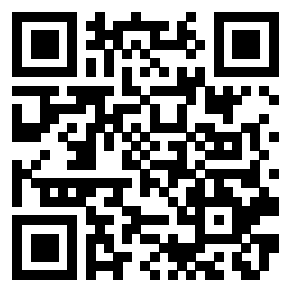

\begin{abstract}
Purpose: This study evaluated the effects of the mixed essential oils containing sweet orange, lavender, and amyris (MEO) on human electroencephalogram (EEG) activity. Methods: EEG activity was recorded by examining the sequence of brain waves of 20 adults, aged from 20 to 30, before and during inhaling the mixed essential oils. Results: MEO showed activity centered on the frontal lobe, which is responsible for higher-order functions against external stimuli, and this result indicated that the oils acted as an intellectual effect. Additional experiments showed that the brain was relaxed and stabilized through a decrease in the absolute slow alpha (ASA) and the relative slow alpha (RSA), a decrease in the absolute beta (AB) and the absolute high beta (AHB), and an increase in the spectral edge frequency $50 \%$ of alpha (ASEF), respectively. Also, the oils induced the awakening states of the brain with a decrease in the absolute alpha (AA) and the absolute theta (AT), and increase of the spectral edge frequency $50 \%$ (SEF50). Furthermore, it was possible to confirm the state of brain immersion through the increase in the absolute fast alpha (AFA), relative fast alpha (RFA), relative mid beta (RMB), ratio of mid beta to theta (RMT), ratio of SMR to theta (RSMT), relative gamma (RG) and the spectral edge frequency 90\% (SEF90). Conclusion: Taken together, these results suggest that the inhaling of MEO affect the brain to be a good condition and improves its concentration ability.
\end{abstract}

Keywords: Aroma, Aromatherapy, Brain wave, Electroencephalography, Essential oil

\section{Introduction}

방향성 식물의 꽃, 열매, 껍질, 잎, 줄기, 뿌리 등 에센스가 함유 되어 있는 재료에 증기나 압력을 가해 추출한 에센셜 오일은(Sim et al., 2016; Yong et al., 2017), 고농도, 지용성의 휘발성 유기 화합물 로 피부와 후각을 통해 인체에 흡수될 수 있다(Choi, 2014; Jung \& Song, 2018). 흡입은 가장 빠른 인체의 흡수 방법으로 대뇌의 변연 계를 통해 두뇌로 전달되어 영향을 준다(Kim et al., 2013).

이러한 에센셜 오일이 뇌에 미치는 영향에 대해 뇌파라는 과학 적인 방법을 사용한 연구가 활발하게 이루어지고 있다(Heo et al., 2017). 라벤더(Torii et al., 1988; Diego et al., 1998; Masago et al., 2000; Min et al., 2001; Sayorwan et al., 2012; Kang et al., 2013), 마조람(Lim, 2011), 로즈(Liu et al., 2013), 로만 카모마일
(Jung \& Lim, 2016)의 이완 효과가 보고되어 있고, 에센셜 오일이 저농도보다는 고농도에서 뇌파 지표 활성의 차이를 보였으며(Kang et al, , 2013), 향의 선호도가 뇌파와 상관관계가 있음이 보고되어 있 다(Min et al., 1999; Min, 2001; Lee \& Kang, 2019).

뇌파(electroencephalogram, $\mathrm{EEG}$ )는 뇌에서 발생하는 전기적인 신호로, 두피 뇌파(scalp $\mathrm{EEG}$ )는 두피에 부착한 전극을 통해 머리 표 면에 형성되는 공간적인 전위차를 측정한 것이다(Kim et al., 2017). 이러한 뇌파는 주파수 범위에 따라 delta파(0.5-4 Hz), theta파(4-8 $\mathrm{Hz})$, alpha파 $(8-14 \mathrm{~Hz})$, beta파 $(14-30 \mathrm{~Hz})$, gamma파 $(30-50 \mathrm{~Hz})$ 로 분류된다. Delta파는 숙면 중에, theta파는 정서적 안정, 수면 상 태, 휴식을 취할 때 나타난다. Alpha파는 두뇌의 안정상태를 나타내 며, beta파는 암산 등의 정신활동, 긴장, 집중을 필요로 할 때, 비교 적 스트레스가 많은 활동적인 정신상태를 나타낸다. Gamma파는 긴 
장 상태, 추리나 판단 등 고도의 복합정신기능 수행 시 나타난다(Kim et al., 2018b).

대뇌는 전두엽(Frontal lobe), 두정엽(Parietal lobe), 측두엽 (Temporal lobe), 후두엽(Occipital lobe)으로 되어 있으며, 전두엽 은 집중, 선택 등의 고도의 인지에 관여하며, 중심 부분인 두정엽은 운동, 감각에 관여하고, 대뇌 양 옆의 측두엽은 청각, 뒤통수에 위치 한 후두엽은 주요 시각 중추로 시각 정보를 처리한다(Kim \& Ryu, 2011; Lee et al., 2016).

본 연구에서는 에센셜 오일 중 스위트 오렌지, 라벤더, 아미리스 를 함유한 mixed essential oil의 향기 흡입이 뇌파에 어떠한 영향을 미치는 지 확인해 보았다. 에센셜 오일은 심신의 상태나 향의 선호 도, 특성, 상호작용 및 주의사항 등을 고려하여 블렌딩 할 수 있으며 (Choi, 2014), 이러한 시너지 블렌딩이 그 효과를 극대화할 수 있다 고 보고되어 있다(Oh et al., 2000; Bae \& Her, 2016).

본 연구에서 사용한 스위트 오렌지, 라벤더, 아미리스 에센셜 오 일의 선정은 효능, 효과와 안전성, 향기 선호도에 중점을 두고 이루 어졌으며, 처방은 탑, 미들, 베이스 노트를 $1: 2: 1$ 로 하였다(Chang et al., 2008). 향기 요법의 장점은 자연 요법으로 정확하게 응용되었 을 때 부작용이 없지만, 에센셜 오일은 그 성질이 다양하고 침투력이 강하기 때문에 민감한 사람에게는 부작용을 일으킬 수 있다(Han \& Choi, 2004).

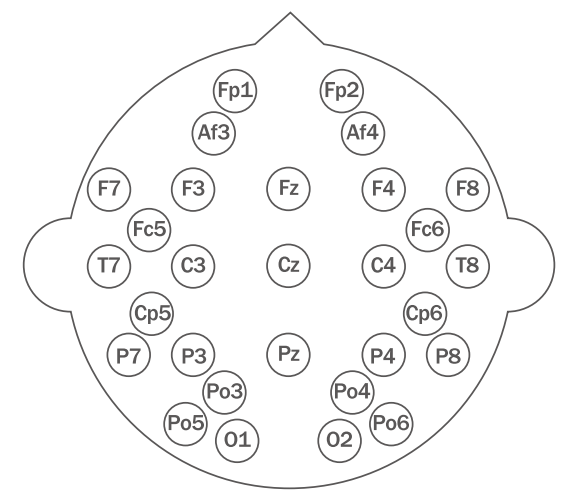

Figure 1. The electrode placement locations according to the international $10-20$ system with modified combinational nomenclature.

Table 1. EEG power spectrum indices used in this study

\begin{tabular}{|c|c|c|c|}
\hline No. & EEG indices & The full name of the EEG power spectrum indices & Wavelength range $(\mathrm{Hz})$ \\
\hline 1 & AT & Absolute theta & $4-8$ \\
\hline 2 & AA & Absolute alpha & $8-13$ \\
\hline 3 & $A B$ & Absolute beta & $13-30$ \\
\hline 4 & AG & Absolute gamma & $30-50$ \\
\hline 5 & ASA & Absolute slow alpha & $8-11$ \\
\hline 6 & AFA & Absolute fast alpha & $11-13$ \\
\hline 7 & ALB & Absolute low beta & $12-15$ \\
\hline 8 & AMB & Absolute mid-beta & $15-20$ \\
\hline 9 & $\mathrm{AHB}$ & Absolute high beta & $20-30$ \\
\hline 10 & RT & Relative theta & $(4-8) /(4-50)$ \\
\hline 11 & RA & Relative alpha & $(8-13) /(4-50)$ \\
\hline 12 & RB & Relative beta & $(13-30) /(4-50)$ \\
\hline 13 & $R G$ & Relative gamma & $(30-50) /(4-50)$ \\
\hline 14 & RSA & Relative slow alpha & $(8-11) /(4-50)$ \\
\hline 15 & RFA & Relative fast alpha & $(11-13) /(4-50)$ \\
\hline 16 & RLB & Relative low beta & $(12-15) /(4 \sim 50)$ \\
\hline 17 & RMB & Relative mid-beta & $(15-20) /(4-50)$ \\
\hline 18 & $\mathrm{RHB}$ & Relative high beta & $(20-30) /(4-50)$ \\
\hline 19 & RST & Ratio of SMR to theta & $(12-15) /(4-8)$ \\
\hline 20 & RMT & Ratio of mid-beta to theta & $(15-20) /(4-8)$ \\
\hline 21 & RSMT & Ratio of SMR $\sim$ mid-beta to theta & $(12-20) /(4-8)$ \\
\hline 22 & RAHB & Ratio of alpha to high beta & $(8-13) /(20-30)$ \\
\hline 23 & SEF50 & Spectral edge frequency $50 \%$ & $4-50$ \\
\hline 24 & SEF90 & Spectral edge frequency $90 \%$ & $4-50$ \\
\hline 25 & ASEF & Spectral edge frequency $50 \%$ of alpha & $8-13$ \\
\hline
\end{tabular}


탑 노트인 스위트 오렌지는 감귤 계열로 광독성[자외선 빛이 존재 할 때 발생하는 피부 반응으로 에센셜 오일의 furocoumarine 성분 (주로 감귤 계열에 함유)이 빛을 더 많이 흡수하는 성질에 의한 것 임] 유발 가능성이 낮아 비교적 안전하며(Choi, 2014), 향기 선호도가 있 다고 보고되어 있다(Baik et al., 1998). 미들 노트에 해당하는 라벤 더는 비교적 널리 활용되는 에센셜 오일로(Kang et al., 2013), 전
신 이완에 도움을 주며, 아이와 노인에게도 안전한 에센셜 오일이다 (Choi, 2014).

베이스 노트는 비교적 안전하면서도 진정 작용이 있는 우디 계 열 에센셜 오일인 세다우드, 패출리, 아미리스 에센셜 오일로(Lee et al., 2019), 우디계열 에센셜 오일의 향기 심미성이 낮은 점을 보완하 기 위해(Park et al., 2001; Oh, 2012), 예비 향기 선호도 조사(서울

Table 2. Significant changes of absolute alpha power spectrum values before and during the inhalation of the mixed essential oils ( $\mathrm{N}=18$ )

\begin{tabular}{|c|c|c|c|c|c|c|}
\hline \multirow{3}{*}{ Site } & \multicolumn{4}{|c|}{ Absolute alpha values (AA) } & \multirow{3}{*}{$t$-test } & \multirow{3}{*}{$p^{*}$} \\
\hline & \multicolumn{2}{|c|}{ Before } & \multicolumn{2}{|c|}{ During } & & \\
\hline & Mean & SD & Mean & SD & & \\
\hline Fp1- Left prefrontal & 30.709 & 22.689 & 26.868 & 19.302 & 3.011 & 0.008 \\
\hline Fp2- Right prefrontal & 34.695 & 26.658 & 30.140 & 22.138 & 2.926 & 0.009 \\
\hline Af3- Left prefrontal & 35.940 & 26.360 & 31.570 & 22.624 & 2.847 & 0.011 \\
\hline Af4- Right prefrontal & 40.935 & 31.196 & 35.732 & 25.962 & 2.873 & 0.011 \\
\hline F7- Left frontal & 21.638 & 15.036 & 19.013 & 12.877 & 3.101 & 0.006 \\
\hline F8- Right frontal & 30.688 & 25.330 & 26.896 & 20.680 & 2.702 & 0.015 \\
\hline F3- Left frontal & 36.903 & 25.264 & 33.346 & 22.200 & 2.267 & 0.037 \\
\hline Fz- Central frontal & 44.458 & 31.697 & 39.774 & 27.103 & 2.489 & 0.023 \\
\hline F4- Right frontal & 43.704 & 32.240 & 39.103 & 27.665 & 2.547 & 0.021 \\
\hline Fc5- Left frontal & 23.837 & 17.403 & 21.398 & 15.000 & 2.428 & 0.027 \\
\hline Fc6- Right frontal & 34.854 & 27.718 & 30.999 & 23.825 & 2.952 & 0.009 \\
\hline
\end{tabular}

Significant difference $(p<0.05)$; SD, standard deviation.

Table 3. Significant changes of absolute slow alpha power spectrum values before and during the inhalation of the mixed essential oils ( $\mathrm{N}=18$ )

\begin{tabular}{|c|c|c|c|c|c|c|}
\hline \multirow{3}{*}{ Site } & \multicolumn{4}{|c|}{ Absolute slow alpha values (ASA) } & \multirow{3}{*}{$t$-test } & \multirow{3}{*}{$p^{*}$} \\
\hline & \multicolumn{2}{|c|}{ Before } & \multicolumn{2}{|c|}{ During } & & \\
\hline & Mean & SD & Mean & SD & & \\
\hline Fp1- Left prefrontal & 25.592 & 21.366 & 21.363 & 17.791 & 3.449 & 0.003 \\
\hline Fp2- Right prefrontal & 28.572 & 24.802 & 24.010 & 20.291 & 3.083 & 0.007 \\
\hline Af3- Left prefrontal & 29.975 & 24.883 & 25.175 & 21.047 & 3.528 & 0.003 \\
\hline Af4- Right prefrontal & 33.905 & 29.168 & 28.376 & 23.973 & 3.244 & 0.005 \\
\hline F7- Left frontal & 18.107 & 14.442 & 15.070 & 12.024 & 3.867 & 0.001 \\
\hline F8- Right frontal & 25.296 & 23.791 & 21.331 & 19.342 & 2.833 & 0.011 \\
\hline F3- Left frontal & 30.739 & 24.167 & 26.598 & 21.048 & 3.146 & 0.006 \\
\hline Fz- Central frontal & 36.786 & 29.877 & 31.539 & 25.316 & 3.150 & 0.006 \\
\hline F4- Right frontal & 35.979 & 30.259 & 30.906 & 25.867 & 3.045 & 0.007 \\
\hline Fc5- Left frontal & 19.964 & 16.882 & 17.047 & 14.310 & 3.258 & 0.005 \\
\hline Fc6-Right frontal & 28.586 & 26.383 & 24.465 & 22.586 & 3.248 & 0.005 \\
\hline T7- Left temporal & 10.648 & 9.482 & 9.431 & 8.385 & 2.125 & 0.049 \\
\hline T8- Right temporal & 20.504 & 18.962 & 18.336 & 17.739 & 2.179 & 0.044 \\
\hline Cz- Central parietal & 40.416 & 34.352 & 36.014 & 29.777 & 2.352 & 0.031 \\
\hline P8- Right parietal & 43.718 & 37.807 & 34.983 & 27.581 & 2.375 & 0.030 \\
\hline
\end{tabular}

Significant difference $(p<0.05)$; SD, standard deviation. 
소재 화장품 $\mathrm{ODM} / \mathrm{OEM}$ 전문기업 $\mathrm{N}$ 사의 20-30대 여성 직원을 대상 으로)를 실시하였고, 최종 스위트 오렌지, 라벤더, 아미리스 에센셜 오일이 함유된 mixed essential oil로 선정하였다(17명 중 12 명 선정, 약 $70 \%$ ).

\section{Methods}

\section{1. 연구대상 및 기간}

연구기간은 2020년 5월부터 1개월간 후각 질환이 없는 20-30대 의 건강한 성인남자 10 명, 여자 10 명 총 20 명을 대상으로 진행했으 며, 약 복용, 수술 이력이 있는 사람은 제외되었다. 뇌파 측정 전 24 시간 동안 감기약 복용 및 술과 담배를 하지 않고 충분한 수면과 휴식 을 취하여 피로를 겪지 않도록 주의를 주었다. 본 연구는 강원대학교 천연향료 실험실에 뇌파 분석을 의뢰하여, 강원대학교 기관생명윤리
위원회의 승인(KWNUIRB-2020-05-001-001)을 받아 진행하였 다.

\section{2. 실험방법}

뇌파 측정은 약 온도 $24^{\circ} \mathrm{C}$, 습도 $50 \%$ 로 유지되는 $32.4 \mathrm{~m}^{2}$ 크기 의 조용한 실험실에서 진행되었다. 뇌파 전극부착은 Figure 1 과 같 은 10-20 국제표준 전극 부착법에 따라 32채널 뇌파 전극이 부착되 었다. 스위트 오렌지, 라벤더, 아미리스 에센셜 오일이 함유된 mixed essential oil이 뇌파에 어떠한 영향을 미치는 지 확인하기 위해, 피험 자가 눈을 감은 상태에서 흡입 전과 흡입 중 각각 $1 \mathrm{~min}$ 간 뇌파를 측 정하고 분석하였다. 흡입 중 뇌파는 $250 \mathrm{~mL}$ 샘플 챔버 내 향료 투입 후, 산소 펌프 시스템을 통해 무취의 공기를 $3 \mathrm{~L} / \mathrm{min}$ 의 유량으로 투 입하여 튜브를 통해 눈을 감은 피험자의 코로부터 $5 \mathrm{~cm}$ 위치에 고정 된 마스크로 $1 \mathrm{~min}$ 간 향기를 맡으면서 뇌파를 측정하였다.

뇌파 측정 결과는 Telescan (LXSMD61; Laxtha Co. Ltd., Korea)

Table 4. Significant changes of relative slow alpha power spectrum values before and during the inhalation of the mixed essential oils $(\mathrm{N}=18)$

\begin{tabular}{|c|c|c|c|c|c|c|}
\hline \multirow{3}{*}{ Site } & \multicolumn{4}{|c|}{ Relative slow alpha values (RSA) } & \multirow{3}{*}{$t$-test } & \multirow{3}{*}{$p^{*}$} \\
\hline & \multicolumn{2}{|c|}{ Before } & \multicolumn{2}{|c|}{ During } & & \\
\hline & Mean & SD & Mean & SD & & \\
\hline Af4- Right prefrontal & 0.379 & 0.139 & 0.346 & 0.132 & 2.331 & 0.032 \\
\hline F4- Right frontal & 0.375 & 0.136 & 0.345 & 0.132 & 2.245 & 0.038 \\
\hline Fc5- Left frontal & 0.351 & 0.137 & 0.321 & 0.139 & 2.363 & 0.030 \\
\hline Fc6- Right frontal & 0.337 & 0.124 & 0.308 & 0.115 & 2.316 & 0.033 \\
\hline P7- Left parietal & 0.384 & 0.156 & 0.342 & 0.138 & 2.439 & 0.026 \\
\hline P8- Right parietal & 0.403 & 0.129 & 0.349 & 0.127 & 2.754 & 0.014 \\
\hline Po5- Left occipital & 0.430 & 0.149 & 0.378 & 0.149 & 2.181 & 0.044 \\
\hline Po6- Right occipital & 0.438 & 0.149 & 0.370 & 0.157 & 2.889 & 0.010 \\
\hline
\end{tabular}

Significant difference $(p<0.05)$; SD, standard deviation.

Table 5. Significant changes of absolute fast alpha power spectrum values before and during the inhalation of the mixed essential oils ( $\mathrm{N}=18$ )

\begin{tabular}{|c|c|c|c|c|c|c|}
\hline \multirow{3}{*}{ Site } & \multicolumn{4}{|c|}{ Absolute fast alpha values (AFA) } & \multirow{3}{*}{$t$-test } & \multirow{3}{*}{$p^{*}$} \\
\hline & \multicolumn{2}{|c|}{ Before } & \multicolumn{2}{|c|}{ During } & & \\
\hline & Mean & SD & Mean & SD & & \\
\hline C4- Right temporal & 9.472 & 6.514 & 11.433 & 9.358 & -2.364 & 0.030 \\
\hline Cp6- Right temporal & 10.843 & 8.311 & 14.595 & 14.889 & -2.243 & 0.039 \\
\hline P7- Left parietal & 7.336 & 5.480 & 9.811 & 8.989 & -2.334 & 0.032 \\
\hline P3- Left parietal & 13.085 & 10.315 & 17.560 & 16.994 & -2.335 & 0.032 \\
\hline Pz- Central parietal & 14.181 & 10.354 & 19.155 & 17.054 & -2.755 & 0.014 \\
\hline P4- Right parietal & 14.926 & 11.764 & 21.147 & 21.127 & -2.604 & 0.019 \\
\hline Po5- Left occipital & 13.973 & 11.475 & 18.423 & 16.977 & -2.569 & 0.020 \\
\hline Po6- Right occipital & 19.613 & 17.267 & 29.227 & 31.789 & -2.489 & 0.023 \\
\hline Po4- Right occipital & 19.091 & 15.841 & 27.440 & 29.985 & -2.232 & 0.039 \\
\hline 01- Left occipital & 14.320 & 11.419 & 19.462 & 17.674 & -2.528 & 0.022 \\
\hline O2- Right occipital & 16.959 & 13.741 & 23.969 & 24.855 & -2.252 & 0.038 \\
\hline
\end{tabular}

Significant difference $(p<0.05)$; SD, standard deviation. 
프로그램을 통하여 데이터 결과가 실시간으로 컴퓨터에 저장되었으 며, 뇌파 기기 QEEE-64FX(LAXTHA Inc., Korea)를 이용하였다. 25 개 지표를 대상으로 뇌파 분석하였으며, 뇌파지표 항목은 Table 1 에 나타내었다.

뇌파지표 항목 중 절대파워는 두피에서 바로 측정되어 사람마다 다른 뇌의 주변 조직(두피, 뼈 분포 등)에 대한 영향을 제외하기 어려 우나, 전체 스펙트럼에서 특정 주파수 영역의 절대파워가 차지하는 비율인 상대파워를 함께 제시하여 뇌파 측정의 개인적 요소가 주는 영향을 보완하였다(Kim et al., 2017).

\section{3. 자료처리 및 분석}

뇌파 분석은 측정에 사용된 Telescan (LXSMD61; Laxtha co. Ltd.) 프로그램을 사용하였다. 향 자극 전과 흡입 중 차이가 자극 제시 후 30 $\mathrm{s}$ 일 때 가장 크다는 연구결과를 근거로(Nam et al., 2000), 1분간 측정 된 피험자의 뇌파 데이터 중 중간 $30 \mathrm{~s}$ 간의 데이터를 추출하여 시계열 분석을 실시하였고, 고속 퓨리에 변환(Fast Fourier transform)을 이용 하여 시계열 데이터를 주파수 영역으로 변환한 파워 스펙트럼 분석법을 이용하였다. 분석 시 피험자 20 명 중 잡파의 혼입을 나타낸 2 명의 데이
터는 제외하였다.

데이터는 통계분석 프로그램인 SPSS (SPSS 12.0 version; SPSS $\mathrm{Inc}$, USA)로 대응 표본 T 검정을 통한 데이터의 평균(mean), 표준편차 (standard deviation)를 나타내었다.

\section{Results and Discussion}

본 연구는 스위트 오렌지, 라벤더, 아미리스 에센셜 오일이 함유된 mixed essential oil(이하 MEO)의 흡입이 뇌파에 어떠한 영향을 미치는 지 25 개의 뇌파 지표를 통해 분석하였다. 그 결과 $\mathrm{AT}, \mathrm{AA}, \mathrm{AB}, \mathrm{ASA}$, AFA, AHB, RG, RSA, RFA, RMB, RMT, RSMT, SEF50, SEF90, $\mathrm{ASEF}$ 지표에서 통계적으로 유의미한 결과가 나타났다.

\section{MEO의 흡입이 뇌파 지표 AA, ASA, RSA에 미치는 영향}

Alpha파는 긴장이완 상태에서 나타나는 뇌파로(Kim \& Ryu, 2011), $\mathrm{MEO}$ 의 흡입은 전두엽 부위의 $\mathrm{AA}$ 지표(absolute alpha power spectrum, at Fp1, Fp2, Af3, Af4, F7, F8, F3, Fz, F4, Fc5, and Fc6 sites, Table 2)

Table 6. Significant changes of relative fast alpha power spectrum values before and during the inhalation of the mixed essential oils ( $N=18$ )

\begin{tabular}{|c|c|c|c|c|c|c|}
\hline \multirow{3}{*}{ Site } & \multicolumn{4}{|c|}{ Relative fast alpha values (RFA) } & \multirow{3}{*}{$t$-test } & \multirow{3}{*}{$p^{*}$} \\
\hline & \multicolumn{2}{|c|}{ Before } & \multicolumn{2}{|c|}{ During } & & \\
\hline & Mean & SD & Mean & SD & & \\
\hline Fp1- Left prefrontal & 0.084 & 0.045 & 0.098 & 0.058 & -2.893 & 0.010 \\
\hline Af3- Left prefrontal & 0.087 & 0.046 & 0.100 & 0.060 & -2.471 & 0.024 \\
\hline F7- Left frontal & 0.077 & 0.036 & 0.091 & 0.050 & -3.034 & 0.007 \\
\hline F3- Left frontal & 0.086 & 0.045 & 0.099 & 0.058 & -2.587 & 0.019 \\
\hline Fc5- Left frontal & 0.081 & 0.037 & 0.094 & 0.050 & -2.717 & 0.015 \\
\hline C3- Left temporal & 0.103 & 0.053 & 0.122 & 0.069 & -2.550 & 0.021 \\
\hline C4- Right temporal & 0.118 & 0.061 & 0.138 & 0.083 & -2.518 & 0.022 \\
\hline Cp5- Left temporal & 0.129 & 0.073 & 0.151 & 0.094 & -2.246 & 0.038 \\
\hline Cp6- Right temporal & 0.136 & 0.068 & 0.172 & 0.114 & -2.690 & 0.015 \\
\hline Cz- Central parietal & 0.103 & 0.062 & 0.122 & 0.084 & -2.539 & 0.021 \\
\hline P7- Left parietal & 0.134 & 0.079 & 0.168 & 0.104 & -2.867 & 0.011 \\
\hline P8- Right parietal & 0.137 & 0.076 & 0.182 & 0.122 & -3.078 & 0.007 \\
\hline P3- Left parietal & 0.152 & 0.091 & 0.178 & 0.113 & -2.132 & 0.048 \\
\hline Pz- Central parietal & 0.144 & 0.088 & 0.177 & 0.119 & -2.624 & 0.018 \\
\hline P4- Right parietal & 0.153 & 0.084 & 0.195 & 0.134 & -2.540 & 0.021 \\
\hline Po5- Left occipital & 0.155 & 0.090 & 0.197 & 0.118 & -2.892 & 0.010 \\
\hline Po6- Right occipital & 0.157 & 0.091 & 0.220 & 0.141 & -3.812 & 0.001 \\
\hline Po4- Right occipital & 0.178 & 0.102 & 0.223 & 0.150 & -2.163 & 0.045 \\
\hline 01- Left occipital & 0.149 & 0.087 & 0.196 & 0.121 & -2.686 & 0.016 \\
\hline O2- Right occipital & 0.164 & 0.095 & 0.214 & 0.139 & -2.817 & 0.012 \\
\hline
\end{tabular}

Significant difference $(p<0.05)$; SD, standard deviation. 
를 통계적으로 유의미하게 감소시켜 뇌의 각성 상태를 확인할 수 있었 다.

Alpha파의 활성은 향기에 노출되는 동안의 긍정적, 부정적인 심리 적 변화에 영향을 받는다고 보고되어 있다. 라벤더 에센셜 오일의 흡입 은 두정엽, 측두엽 부위에서 alpha1 (8-10 Hz, slow alpha파)의 활성 을 감소시켰는데, 이는 편안한 상태와 높은 상관관계가 있음이 보고되 어 있다(Sowndhararajan \& Kim, 2016; Lee et al., 2019). MEO의 흡입 은 전두엽, 측두엽, 두정엽 부위의ASA 지표(absolute slow alpha power spectrum, at Fp1, Fp2, Af3, Af4, F7, F8, F3, Fz, F4, Fc5, Fc6, T7, T8, $\mathrm{Cz}$, and P8 sites, Table 3), 그리고 전두엽, 두정엽, 후두엽 부위의RSA지
표(Relative slow alpha power spectrum, at Af4, F4, Fc5, Fc6, P7, P8, Po5, and Po6 sites, Table 4)를 통계적으로 유의미하게 감소시켜 편안한 상태임을 확인할 수 있었다.

\section{MEO의 흡입이 뇌파 지표 AFA, RFA, ASEF에 미치는 영향}

$\mathrm{MEO}$ 의 흡입은 측두엽, 두정엽, 후두엽 부위의AFA 지표(Absolute fast alpha power spectrum, at C4, Cp6, P7, P3, Pz, P4, Po5, Po6, Po4, O1 and O2 sites, Table 5), 전두엽, 측두엽, 두정엽, 후두엽 부위의 RFA 지표(relative fast alpha power spectrum, Fp1, Af3, F7, F3, Fc5, C3, Cz, C4, Cp5, Cp6, P7, P8, P3, Pz, P4, Po5, Po6, Po4, O1, and

Table 7. Significant changes of spectral edge frequency $\mathbf{5 0 \%}$ of alpha power spectrum values before and during the inhalation of the mixed essential oils

\begin{tabular}{|c|c|c|c|c|c|c|}
\hline \multirow{3}{*}{ Site } & \multicolumn{4}{|c|}{ ASEF (Spectral edge frequency $50 \%$ of alpha power spectrum values) } & \multirow{3}{*}{$t$-test } & \multirow{3}{*}{$p^{*}$} \\
\hline & \multicolumn{2}{|c|}{ Before } & \multicolumn{2}{|c|}{ During } & & \\
\hline & Mean & SD & Mean & SD & & \\
\hline Fp1- Left prefrontal & 10.103 & 0.565 & 10.213 & 0.519 & -3.552 & 0.002 \\
\hline Fp2- Right prefrontal & 10.100 & 0.569 & 10.179 & 0.541 & -2.413 & 0.027 \\
\hline Af3- Left prefrontal & 10.122 & 0.557 & 10.210 & 0.538 & -2.938 & 0.009 \\
\hline Af4- Right prefrontal & 10.096 & 0.565 & 10.203 & 0.558 & -2.986 & 0.008 \\
\hline F8- Right frontal & 10.078 & 0.575 & 10.188 & 0.560 & -2.836 & 0.011 \\
\hline F3- Left frontal & 10.113 & 0.559 & 10.218 & 0.538 & -2.545 & 0.021 \\
\hline Fz- Central frontal & 10.105 & 0.565 & 10.201 & 0.539 & -2.127 & 0.048 \\
\hline F4- Right frontal & 10.096 & 0.569 & 10.213 & 0.550 & -2.788 & 0.013 \\
\hline Fc5- Left frontal & 10.125 & 0.552 & 10.235 & 0.523 & -2.802 & 0.012 \\
\hline Fc6- Right frontal & 10.117 & 0.581 & 10.200 & 0.549 & -2.303 & 0.034 \\
\hline Cz- Central parietal & 10.203 & 0.637 & 10.369 & 0.703 & -2.267 & 0.037 \\
\hline C4- Right temporal & 10.317 & 0.683 & 10.457 & 0.715 & -2.296 & 0.035 \\
\hline Cp6- Right temporal & 10.445 & 0.659 & 10.642 & 0.758 & -2.897 & 0.010 \\
\hline P7- Left parietal & 10.367 & 0.507 & 10.510 & 0.498 & -2.335 & 0.032 \\
\hline P8- Right parietal & 10.364 & 0.537 & 10.535 & 0.572 & -3.546 & 0.002 \\
\hline P4- Right parietal & 10.557 & 0.731 & 10.705 & 0.768 & -2.614 & 0.018 \\
\hline Po5- Left occipital & 10.459 & 0.478 & 10.603 & 0.500 & -2.149 & 0.046 \\
\hline Po6- Right occipital & 10.429 & 0.527 & 10.629 & 0.542 & -4.063 & 0.001 \\
\hline Po4- Right occipital & 10.625 & 0.694 & 10.778 & 0.670 & -2.349 & 0.031 \\
\hline 02- Right occipital & 10.493 & 0.542 & 10.707 & 0.579 & -2.706 & 0.015 \\
\hline
\end{tabular}

Significant difference $(p<0.05)$; SD, standard deviation.

Table 8. Significant changes of absolute beta power spectrum values before and during the inhalation of the mixed essential oils $(\mathrm{N}=18)$

\begin{tabular}{|c|c|c|c|c|c|c|}
\hline \multirow{3}{*}{ Site } & \multicolumn{4}{|c|}{ Absolute beta values (AB) } & \multirow{3}{*}{$t$-test } & \multirow{3}{*}{$p^{*}$} \\
\hline & \multicolumn{2}{|c|}{ Before } & \multicolumn{2}{|c|}{ During } & & \\
\hline & Mean & SD & Mean & SD & & \\
\hline Af4- Right prefrontal & 15.172 & 8.677 & 14.440 & 8.066 & 2.473 & 0.024 \\
\hline F7- Left frontal & 10.526 & 7.858 & 9.656 & 6.437 & 2.133 & 0.048 \\
\hline F8- Right frontal & 14.609 & 8.256 & 13.384 & 6.588 & 2.328 & 0.033 \\
\hline
\end{tabular}

Significant difference $(p<0.05)$; SD, standard deviation. 
O2 sites, Table 6), ASEF 지표(spectral edge frequency $50 \%$ of alpha spectrum band, at Fp1, Fp2, Af3, Af4, F8, F3, Fz, F4, Fc5, Fc6, Cz, $\mathrm{C} 4, \mathrm{Cp} 6, \mathrm{P} 7, \mathrm{P} 8, \mathrm{P} 4, \mathrm{Po} 5, \mathrm{Po} 6, \mathrm{Po} 4$ and $\mathrm{O} 2$ sites, Table 7)를 통계적 으로 유의미하게 증가시켰다. 편안한 상태에서 몰입하여 창의력을 나타 내는AFA, RFA 지표의 증가와(Kim et al., 2018a), 쾌적성을 나타내는 $\mathrm{ASEF}$ 지표의 증가를 통해 전체적으로 빠른 alpha파가 많아 뇌가 쾌적 한 상태임을 확인할 수 있었다. 일반적으로 알파파의 활성이 높을수록 스트레스 상태의 감소 수준과 높은 상관관계가 있다고 보고되어 있으며 (Sowndhararajan \& Kim, 2016), $\mathrm{MEO}$ 의 흡입은 스트레스 완화에 도움 을 줄 것으로 사료된다.

\section{3. $\mathrm{MEO}$ 의 흡입이 뇌파 지표 $\mathrm{AB}, \mathrm{AHB}$ 에 미치는 영향}

Beta파는 개안, 불안, 암산 등의 정신활동이나 청각, 피부 자극, 통증 등의 긴장, 집중을 필요로 할 때 대뇌 피질에 광범위하게 나타나며, 비 교적 스트레스가 많은 활동적인 정신 상태를 나타내는 뇌파이다(Kim et al., 2018b). $\mathrm{MEO}$ 의 흡입은 전두엽 부위의 $\mathrm{AB}$ 지표(absolute beta power spectrum, at Af4, F7, and F8 sites, Table 8), AHB 지표(absolute high beta power spectrum, at Af4 and F3 sites, Table 9)를 통계적으로
유의미하게 감소시켰으며, 이는 뇌의 긴장, 스트레스 상태의 완화를 나 타낸다.

\section{4. $\mathrm{MEO}$ 의 흡입이 뇌파 지표 RMB에 미치는 영향}

Mid beta파는 고도의 인지과정이나 초 집중 상태에서 나타나며 비 교적 정신 부하가 높은 사고활동을 수행할 때 나타나는데(Kim et al, $2018 \mathrm{~b}), \mathrm{MEO}$ 의 흡입은 전두엽 부위의 $\mathrm{RMB}$ 지표(relative mid beta power spectrum, at Fp1, Fp2, Af3, Af4, F7, F3, and Fc5 sites, Table 10)를 통계적으로 유의미하게 증가시켰다. 이는 고도로 집중하고 있는 상태임을 나타낸다.

\section{MEO의 흡입이 뇌파 지표 AT에 미치는 영향}

Theta파의 변화는 주의력이나 인지부하의 수정을 반영하며, 스 피어민트의 향기가 theta파의 활성을 감소시켰음이 보고되어 있다 (Sowndhararajan \& Kim, 2016). MEO의 흡입은 전두엽 부위의 AT지 표(absolute theta power spectrum, at Fp1 site, Table 11)를 통계적으로 유의미하게 감소시켰다. 얕은 수면상태, 혹은 깊은 명상을 할 때 주로 나타나는 AT 지표의 감소는 뇌의 각성을 의미한다(Kim et al., 2018a).

Table 9. Significant changes of absolute high beta power spectrum values before and during the inhalation of the mixed essential oils

\begin{tabular}{|c|c|c|c|c|c|c|}
\hline \multirow{3}{*}{ Site } & \multicolumn{4}{|c|}{ Absolute high beta values (AHB) } & \multirow{3}{*}{$t$-test } & \multirow{3}{*}{$p^{*}$} \\
\hline & \multicolumn{2}{|c|}{ Before } & \multicolumn{2}{|c|}{ During } & & \\
\hline & Mean & SD & Mean & SD & & \\
\hline Af4- Right prefrontal & 6.905 & 5.156 & 6.328 & 4.462 & 2.297 & 0.035 \\
\hline F3- Left frontal & 6.406 & 3.742 & 5.981 & 3.363 & 2.484 & 0.024 \\
\hline
\end{tabular}

Significant difference $(p<0.05)$; SD, standard deviation.

Table 10. Significant changes of relative mid beta power spectrum values before and during the inhalation of the mixed essential oils $(\mathrm{N}=18)$

\begin{tabular}{|c|c|c|c|c|c|c|}
\hline \multirow{3}{*}{ Site } & \multicolumn{4}{|c|}{ Relative mid beta values (RMB) } & \multirow{3}{*}{$t$-test } & \multirow{3}{*}{$p^{*}$} \\
\hline & \multicolumn{2}{|c|}{ Before } & \multicolumn{2}{|c|}{ During } & & \\
\hline & Mean & SD & Mean & SD & & \\
\hline Fp1- Left prefrontal & 0.072 & 0.032 & 0.078 & 0.032 & -2.424 & 0.027 \\
\hline Fp2- Right prefrontal & 0.075 & 0.032 & 0.083 & 0.034 & -2.804 & 0.012 \\
\hline Af3- Left prefrontal & 0.078 & 0.033 & 0.084 & 0.035 & -2.277 & 0.036 \\
\hline Af4- Right prefrontal & 0.077 & 0.032 & 0.084 & 0.034 & -2.961 & 0.009 \\
\hline F7- Left frontal & 0.080 & 0.027 & 0.086 & 0.030 & -2.457 & 0.025 \\
\hline F3- Left frontal & 0.078 & 0.029 & 0.087 & 0.036 & -3.103 & 0.006 \\
\hline Fc5- Left frontal & 0.081 & 0.026 & 0.091 & 0.032 & -3.845 & 0.001 \\
\hline
\end{tabular}

Significant difference $(p<0.05)$; SD, standard deviation.

Table 11. Significant changes of absolute theta power spectrum values before and during the inhalation of the mixed essential oils ( $N=18$ )

\begin{tabular}{|c|c|c|c|c|c|c|}
\hline \multirow{3}{*}{ Site } & \multicolumn{4}{|c|}{ Absolute theta values (AT) } & \multirow{3}{*}{$t$-test } & \multirow{3}{*}{$p^{*}$} \\
\hline & \multicolumn{2}{|c|}{ Before } & \multicolumn{2}{|c|}{ During } & & \\
\hline & Mean & $\mathrm{SD}$ & Mean & SD & & \\
\hline Fp1- Left prefrontal & 16.105 & 13.880 & 14.267 & 13.492 & 2.282 & 0.036 \\
\hline
\end{tabular}

Significant difference $(p<0.05)$; SD, standard deviation. 
6. MEO의 흡입이 뇌파 지표 RG, RMT, RSMT, SEF50, SEF90에 미치는 영향

$\mathrm{MEO}$ 의 흡입은 전두엽 부위의RMT 지표(ratio of mid beta to theta, at F3 and Fc5 sites, Table 12), 전두엽, 측두엽 부위 의 RSMT 지표(ratio of SMR-mid beta to theta, at Fc5 and T7 sites, Table 13), 전두엽 부위의 $\mathrm{RG}$ 지표(relative gamma power spectrum, at Fp1 and Af3 sites, Table 14), SEF90지표(spectral edge frequency 90\%, at Fp1 and Af3 sites, Table 15)를 통계적으 로 유의미하게 증가시켰다. 계산이나 암산같이 한 가지 주제에 집 중할 때 나타나는RMT지표, 집중상태를 객관적으로 확인할 수 있는 RSMT지표, 주의 집중 또는 지각 교환이 활발하게 이루어질 때 발생 하는RG 지표, 정신부하나 스트레스 하에서 나타나는SEF90지표의 증가는 뇌의 집중, 몰입 상태를 의미한다(Kim et al., 2018a).

Theta파의 감소, RFA, RG, SEF50 (spectral edge frequency
$50 \%$ ) 지표의 증가를 통해, 산조인(Zizyphus jujuba mill.)의 씨앗 에서 추출한 에센셜 오일이 뇌의 주의력과 이완 상태를 증가시킨다 고 보고되어 있다(Sowndhararajan \& Kim, 2016). $\mathrm{MEO}$ 의 흡입 또 한 앞서 언급한 바와 같이 theta파를 감소시키고, $\mathrm{RFA}, \mathrm{RG}$, 그리고 SEF50 (at Fp1, Af3, and F3 sites, Table 16) 지표를 증가시켜, 뇌 가 편안하면서도 주의집중력이 증가한 상태임을 확인할 수 있었다.

\section{MEO의 흡입 효과}

$\mathrm{MEO}$ 의 흡입은 전체적으로 전두엽 부위를 중심으로 활성을 보임 을 확인할 수 있었다. 전두엽의 한 부분으로 후각정보를 받아들이는 부위로 알려진 안와 전두 피질(orbitofrontal cortex)은 후각 정보의 식별과 확인 기능을 담당한다. 측두엽은 향 자극을 생리적 특징으로 받아들이는 반면, 전두엽은 향 자극에 대해 그 적절함을 측정하는 등 의 고차원적 기능을 담당한다(Kang et al, , 2002). 이는 MEO의 흡입

Table 12. Significant changes of ratio of mid beta to theta before and during the inhalation of the mixed essential oils

\begin{tabular}{|c|c|c|c|c|c|c|}
\hline \multirow{3}{*}{ Site } & \multicolumn{4}{|c|}{ Ratio of mid beta to theta (RMT) } & \multirow{3}{*}{$t$-test } & \multirow{3}{*}{$p^{*}$} \\
\hline & \multicolumn{2}{|c|}{ Before } & \multicolumn{2}{|c|}{ During } & & \\
\hline & Mean & SD & Mean & SD & & \\
\hline F3- Left frontal & 0.354 & 0.172 & 0.416 & 0.217 & -2.826 & 0.012 \\
\hline Fc5- Left frontal & 0.396 & 0.168 & 0.477 & 0.260 & -2.712 & 0.015 \\
\hline
\end{tabular}

Significant difference $(p<0.05)$; SD, standard deviation.

Table 13. Significant changes of ratio of (SMR-mid beta) to theta before and during the inhalation of the mixed essential oils

\begin{tabular}{|c|c|c|c|c|c|c|}
\hline \multirow{3}{*}{ Site } & \multicolumn{4}{|c|}{ Ratio of (SMR-mid beta) to theta (RSMT) } & \multirow{3}{*}{$t$-test } & \multirow{3}{*}{$p^{*}$} \\
\hline & \multicolumn{2}{|c|}{ Before } & \multicolumn{2}{|c|}{ During } & & \\
\hline & Mean & SD & Mean & SD & & \\
\hline Fc5- Left frontal & 0.768 & 0.456 & 0.896 & 0.606 & -2.684 & 0.016 \\
\hline T7- Left temporal & 1.126 & 0.732 & 1.309 & 0.946 & -2.264 & 0.037 \\
\hline
\end{tabular}

Significant difference $(p<0.05)$; SD, standard deviation.

Table 14. Significant changes of relative gamma power spectrum values before and during the inhalation of the mixed essential oils ( $\mathrm{N}=18$ )

\begin{tabular}{|c|c|c|c|c|c|c|}
\hline \multirow{3}{*}{ Site } & \multicolumn{4}{|c|}{ Relative gamma values (RG) } & \multirow{3}{*}{$t$-test } & \multirow{3}{*}{$p^{*}$} \\
\hline & \multicolumn{2}{|c|}{ Before } & \multicolumn{2}{|c|}{ During } & & \\
\hline & Mean & SD & Mean & SD & & \\
\hline Fp1- Left prefrontal & 0.078 & 0.044 & 0.091 & 0.059 & -2.223 & 0.040 \\
\hline Af3- Left prefrontal & 0.068 & 0.039 & 0.079 & 0.050 & -2.222 & 0.040 \\
\hline
\end{tabular}

Significant difference $(p<0.05)$; SD, standard deviation.

Table 15. Significant changes of spectral edge frequency $90 \%$ before and during the inhalation of the mixed essential oils

\begin{tabular}{|c|c|c|c|c|c|c|}
\hline \multirow{3}{*}{ Site } & \multicolumn{4}{|c|}{ SEF90 (Spectral edge frequency 90\%) } & \multirow{3}{*}{$t$-test } & \multirow{3}{*}{$p^{*}$} \\
\hline & \multicolumn{2}{|c|}{ Before } & \multicolumn{2}{|c|}{ During } & & \\
\hline & Mean & SD & Mean & SD & & \\
\hline Fp1- Left prefrontal & 25.669 & 7.733 & 27.020 & 8.485 & -2.194 & 0.042 \\
\hline Af3- Left prefrontal & 24.362 & 7.242 & 25.640 & 8.076 & -2.196 & 0.042 \\
\hline
\end{tabular}

Significant difference $(p<0.05)$; SD, standard deviation. 
Table 16. Significant changes of spectral edge frequency $\mathbf{5 0 \%}$ before and during the inhalation of the mixed essential oils $(\mathrm{N}=\mathbf{1 8})$

\begin{tabular}{|c|c|c|c|c|c|c|}
\hline \multirow{3}{*}{ Site } & \multicolumn{4}{|c|}{ SEF50 (Spectral edge frequency $50 \%$ ) } & \multirow{3}{*}{$t$-test } & \multirow{3}{*}{$p^{*}$} \\
\hline & \multicolumn{2}{|c|}{ Before } & \multicolumn{2}{|c|}{ During } & & \\
\hline & Mean & SD & Mean & SD & & \\
\hline Fp1- Left prefrontal & 10.212 & 1.220 & 10.608 & 1.299 & -2.814 & 0.012 \\
\hline Af3- Left prefrontal & 10.269 & 1.186 & 10.500 & 1.247 & -2.512 & 0.022 \\
\hline F3- Left frontal & 10.230 & 1.099 & 10.534 & 1.272 & -2.306 & 0.034 \\
\hline
\end{tabular}

Significant difference $(p<0.05)$; SD, standard deviation.

이 외부 자극에 대한 고차원적 기능을 담당하는 전두엽 영역과 관련 되어 있음을 확인할 수 있다.

종합하면, $\mathrm{MEO}$ 의 흡입은 편안한 상태로 뇌의 각성과 몰입을 유 도하면서, 아로마테라피의 기본적인 효과인 스트레스 완화에 효과가 있음을 확인할 수 있었다.

\section{Conclusion}

본 연구는 20-30대 성인남녀 총 20명을 대상으로, 스위트 오렌 지, 라벤더, 아미리스 에센셜 오일이 함유된 mixed essential oil의 흡입 전과 흡입 중 뇌파 변화를 25 개의 뇌파 지표를 통해 살펴보았 다. 그 결과 $\mathrm{AT}, \mathrm{AA}, \mathrm{AB}, \mathrm{ASA}, \mathrm{AFA}, \mathrm{AHB}, \mathrm{RG}, \mathrm{RSA}, \mathrm{RFA}, \mathrm{RMB}$, $\mathrm{RMT}, \mathrm{RSMT}, \mathrm{SEF} 50, \mathrm{SEF} 90, \mathrm{ASEF}$ 지표에서 통계적으로 유의미한 결과가 나타났다.

Absolute slow alpha (ASA), relative slow alpha (RSA)의 감소, absolute beta(AB)와 absolute high beta( $\mathrm{AHB})$ 의 감소를 통해 뇌가 편안한 상태임을 확인할 수 있었으며, 쾌적성을 나타내는 spectral edge frequency $50 \%$ of alpha (ASEF)의 증가를 통해 전체적으로 쾌 적한 상태임을 확인할 수 있었다.

긴장이완 상태에서 나타나는 absolute alpha (AA)의 감소, 얕 은 수면상태, 혹은 깊은 명상을 할 때 주로 나타나는 absolute theta (AT)의 감소, 뇌파 활성도와 각성을 확인할 수 있는 spectral edge frequency $50 \%$ (SEF50)의 증가를 통해 뇌가 각성된 상태임을 확인 할 수 있었다(Shin \& Kim, 2018).

편안하면서도 몰입할 때 발생하는 absolute fast alpha (AFA), relative fast alpha (RFA)와 정신부하가 높은 활동 시 나타나는 relative mid beta (RMB)의 증가, 집중 상태를 확인할 수 있는 ratio of mid beta to theta (RMT), ratio of SMR to theta (RSMT), 주의 집중이 이루어질 때 발생하는 relative gamma $(\mathrm{RG})$, 정신부하나 스 트레스 하에서 나타나는 spectral edge frequency $90 \%$ (SEF90)의 증가를 통해 뇌가 고도로 몰입하고 있는 상태임을 확인할 수 있었다.

스위트 오렌지, 라벤더, 아미리스 에센셜 오일이 함유된 mixed essential oil의 흡입은 전두엽 부위를 중심으로 활성을 보임을 확인 할 수 있었으며, 이는 감성 효과가 아닌 외부 자극에 대한 고차원적
기능을 담당하는 전두엽과 관련된 지성 효과로 작용했음을 확인할 수 있었다.

따라서 본 연구에서 스위트 오렌지, 라벤더, 아미리스 오일이 함유 된 mixed essential oil을 흡입하면, 흡입 전에 비해 뇌를 편안하게 하 면서도 각성, 집중 효과를 주는 것으로 사료된다.

This work is parts of the Yerin Je's M. S. thesis at the Konkuk University, Seoul, Korea.

\section{Acknowledgements}

본 연구는 (주)나우코스의 지원을 통해 수행되었으며, 뇌파 분 석에 도움을 주신 강원대학교 천연향료 실험실에 감사의 말씀을 드립니다.

\section{Author's contribution}

YJ, HR and JC designed this study together. YJ, SA and SB analyzed the data and wrote the manuscript. All authors read and confirmed the final version of manuscript.

\section{Author details}

Yerin Je (Senior Research Engineer), Research Center, Nowcos co., Ltd., A-1004, BYC Highcity 131, Gasan digital 1-ro, Geumcheon-gu, Seoul, Korea; Sungkwan An (Professor), Department of Cosmetics Engineering, Konkuk University, Seoul 05029, Korea; Hyangseon Ro (President CEO), Research Center, Nowcos co., Ltd., A-1004, BYC Highcity 131, Gasan digital 1-ro, Geumcheon-gu, Seoul, Korea; Jawun Cho (Director), Research Center, Nowcos co., Ltd., A-1004, BYC Highcity 131, Gasan digital 1-ro, Geumcheon-gu, Seoul, Korea; Seunghee Bae (Professor); Department of Cosmetics Engineering, Konkuk University, Seoul 05029, Korea. 


\section{References}

Bae IL, Her MH. The effects of essential oil inhalation on the stress and sympathetic nerve activity. The Korean Journal of Stress Research, 24: 75-83, 2016.

Baik EJ, Lee YY, Lee BH, Moon CH, Lee SH, Han HC. Emotional changes of EEG and autonomic response by olfactory stimuli with orange and valeric acid. Korean Journal of the Science of Emotion \& Sensibility, 1: 105-111, 1998.

Lee EJ, Kim YS, Kim MS, Kim JS, Ryu HJ, Moon JS, Park MJ, Bae GA, Cha HY. The complete guide to aromatherapy 3rd edition Vol.I Foundation \& Materia Medica. Korean Association of Essential Oil, Aroma \& APA, Seoul, pp221 223, 2019. (Battaglia S. 2018).

Chang SB, Chu SH, Kim YI, Yun SH. The effects of aroma inhalation on sleep and fatigue in night shift nurses. Korean Journal of Adult Nursing, 20: 941-949, 2008.

Choi SW. Essential aromatherapy. Euihakseowon, Seoul, pp3181, 2014.

Diego MA, Jones NA, Field T, Hernandez-Reif M, Schanberg S, Kuhn C, McAdam V, Galamaga R, Galamaga M. Aromatherapy positively affects mood, EEG patterns of alertness and math computations. The International Journal of Neuroscience, 96: 217-224, 1998.

Han MR, Choi JS. A study of stratum corneum troubles by nature lays on treatments bergamot \& grapefruit essential oil. Journal of the Korean Society of Cosmetology, 10: 202 208, 2004

Heo SH, Yoo SM, Shim JY. Effects of inhalation of two kinds of blended essential oils based on parts of a plant on autonomic nervous system. Journal of the Korean Society of cosmetology, 23: 461-474, 2017.

Jung $\mathrm{SH}$, Lim $\mathrm{MH}$. The effect of roman chamomile inhalation on autonomic nerve system in middle aged women. Journal of the Korean Society of Cosmetology, 22: 636643, 2016.

Kang IH, Min BC, Jin SH, Jeon KJ, Seng EJ, Kim CJ. The relationship between EEG responses and olfactory-related occupations. Korean Journal of the Science of Emotion \& Sensibility, 2002: 128-131, 2002.

Kang SY, Kim MK, Ryu HW. Influence of the concentration of lavender oil on brain activity. Asian Journal of Beauty and Cosmetology, 11: 1099-1107, 2013.

Kim DW, Kim MS, Kim SP, Park YM, Park JY, Bae KY, Lee SH,
Lee JW, Lim CH, Jeon YH, Jin SH, Chae JH, Hwang HJ. Understanding and application of EEG. Hakjisa, Seoul, pp7-82, 2017.

Kim MJ, Song J, Sowndhararajan K, Kim SM. Brain wave response to bottle color of herbicides and non-selective herbicides in Korea. Weed \& Turfgrass Science, 7: 130139, 2018a.

Kim MJ, Yang BH, Kim SM. Effect of fragrance of weeds on the behavior of consumers. Weed \& Turfgrass Science, 7: 98110, 2018b.

Kim MK, Ryu HW. The potentiality of color preference analysis by EEG. Korean Journal of the science of Emotion \& sensibility, 14: 311-320, 2011.

Kim SM, Kim KL, Kim L, Lim BI, Kim SO, Kim HN, Mo JH, Yun MS, Jang MJ, Ha MH. The theory and practice aromatherapy. Jungmunkag, Seoul, pp3-69, 2013.

Lee HA, Kang SJ. The effects of aromatherapy on stress, fatigue and heart rate variability among nursing students in clinical training. The Journal of Learner-Centered Curriculum and Instruction, 19: 1415-1432, 2019.

Lee HJ, Shin DI, Shin DK. A research on the emotion classification and precision improvement of EEG (Electroencephalogram) data using machine learning algorithm. Journal of Internet Computing and Services, 20: 27-36, 2019.

Lee KJ, Lee SY, Park SY, Jang SR, Kang CK. EEG study for the effects of mouth breathing on brain functions. Science of Emotion \& Sensibility, 19: 199-126, 2016.

Lim MH. Effect of inhalation of Origanum majorana essential oil on brain waves. Journal of The Korean Society of cosmetology, 17: 953-961, 2011.

Liu CW, Wang CS, Chuang KJ, Lo CC, Chen CT. Electroencephalographic study of essential oils for stress relief. Applied Mechanics and Materials, 437: 1085-1088, 2013.

Nam KD, Min BC, Chung SC, Lee DH, Min BW, Kim YN, Kim CJ, Kim JS. Time analysis of EEG by essential oils stimuli. Korean. Journal of the science of Emotion \& Sensibility, 2000: 44-47, 2000.

Masago R, Matsuda T, Kikuchi Y, Miyazaki Y, Iwanaga K, Harada $\mathrm{H}$, Katsuura T. Effects of inhalation of essential oils on EEG activity and sensory evaluation. Journal of Physiological Anthropology and Applied Human Science, 19: 35-42, 2000. 
Min BC, Chung SC, Kim SG, Oh JY, Kim HJ, Kim SJ, Kim YN, Shin JS, Min BW, Kim CJ, et al. The assessment of odor using EEG and autonomic responses. Korean Journal of the science of Emotion \& sensibility, 2: 1-10, 1999.

Min BC. Psycho-physiological effect of odors on human being. Journal of the Korean Society for Precision Engineering, 18: 46-53, 2001.

Oh HK, Choi JY, Chun KK, Lee JS, Park DK, Choi SD, Chun TI. A study for antistress and arousal effects and the difference of its effectiveness among three aromatic synergic blending oils. Stress, 8: 9-24, 2000.

Oh YK. The classification and sensitivity analysis of aroma essential oil based on odors evaluation. Journal of The Korean Society of Cosmetology, 18: 237-244, 2012.

Park MK, Chong HY, Lee KH, Choi J, Lee BH, Sohn JH. Psychological structure and ANS response by odor induced emotion. Science of Emotion and Sensibility, 4: 39-45, 2001.

Sayorwan W, Siripornpanich V, Piriyapunyaporn T,
Hongratanaworakit T, Kotchabhakdi N, Ruangrungsi N. The effects of lavender oil inhalation on emotional states, autonomic nervous system, and grain electrical activity. Journal of the Medical Association of Thailand, 95: 598606, 2012.

Shin DJ, Kim JY. A study of response characteristics to color design of Waldorf school classrooms using EEG analysis. Archives of Design Research, 31: 161-174, 2018.

Sowndhararajan K, Kim S. Influence of fragrances on human psychophysiological activity: with special reference to human electroencephalographic response. Scientia Pharmaceutica, 84: 724-752, 2016.

Torii S, Fukuda H, Kanemoto H, Miyauchi R, Hamauzu Y, Kawasaki M. Contingent negative variation (CNV) and the psychological effects of odour. In Perfumery: The Psychology and Biology of Fragrance, van Toller S, Dodd GH(Eds.). Springer, Dordrent, London, UK, pp107-120, 1988. 


\section{국문초록}

\section{Sweet Orange, Lavender, Amyris Essential Oil을 함유한 Mixed Essential Oil향기 가 뇌파에 미치는 영향}

제예린 ${ }^{1,2}$, 안성관 ${ }^{1}$, 노향선 $^{2}$, 조자운 $^{2}$, 배승희 $^{1 *}$

${ }^{1}$ 건국대학교 화장품공학과, 서울, 한국

${ }^{2}$ (주)나우코스 기술연구소, 서울, 한국

목적: 스위트 오렌지, 라벤더, 아미리스 에센셜 오일이 함유된 mixed essential oil흡입 시 인간의 뇌파(EEG)에 미치는 영향을 연구 하고자 한다. 방법: 20-30대 성인남녀 총 20명을 대상으로 흡입 전과 흡입 중의 뇌파를 측정하였다. 결과: 스위트 오렌지, 라벤더, 아미리스 에센셜 오일이 함유된 mixed essential oil은 외부 자극에 대한 고차원적 기능을 담당하는 전두엽 부위를 중심으로 활성을 보여, 지성 효과로 작용했음을 확인할 수 있었다. 향기 흡입 시 absolute slow alpha (ASA), relative slow alpha (RSA), absolute beta (AB), absolute high beta (AHB)의 감소, spectral edge frequency $50 \%$ of alpha (ASEF)지표가 증가하였는데, 이를 통해서 뇌가 편 안해지고 쾌적해진다는 것을 알 수 있었다. 또한 absolute alpha (AA), absolute theta (AT)의 감소, spectral edge frequency $50 \%$ (SEF50)의 증가를 통해 뇌의 각성 상태를 확인할 수 있었다. Absolute fast alpha (AFA), relative fast alpha (RFA), relative mid beta (RMB), ratio of mid beta to theta (RMT), ratio of SMR to theta (RSMT), relative gamma (RG), spectral edge frequency $90 \%$ (SEF90)의 증가를 통해 뇌의 몰입 상태를 확인할 수 있었다. 결론: 결론적으로 스위트 오렌지, 라벤더, 아미리스 에센셜 오일이 함 유된 mixed essential oil의 향기는 뇌가 쾌적하면서도 각성된 상태로, 집중력에 영향을 미치는 것으로 사료된다.

핵심어: 향기, 향기 요법, 뇌파, 뇌파검사, 에센셜 오일

본 연구는 (주)나우코스의 지원을 통해 수행되었으며, 뇌파 분석에 도움을 주신 강원대학교 천연향료 실험실에 감사의 말씀을 드립 니다.

\section{참고문헌}

강승연, 김민경, 류희욱. 라벤더 오일의 농도 변화가 뇌 활성에 미치는 영향. 대한피부미용학회지, 11: 1099-1107, 2013.

강인형, 민병찬, 진승현, 전광진, 성은정, 김철중. 천연향 자극에 대한 향 관련 종사자들의 $\mathrm{EEG}$ 반응. 한국감성과학회지, 2002: 128-131, 2002.

김도원, 김명선, 김성필, 박영민, 박진영, 배경열, 이승환, 이재원, 임창환, 전양환, 진승현, 채정호, 황한정. 뇌파의 이해 와 응용. 학지사. 서울, $\mathrm{pp} 7-82,2017$.

김민경, 류희욱. 뇌파 분석을 통한 색상의 선호도 분석 가능성. 한국감성과학회지, 14: 311-320, 2011.

김민주, 송지은, Sowndhararajan Kandhararajan, 김성문. 제초제 포장지 색상이 소비자들의 뇌파에 미치는 영향. 한국

잡초학회한국잔디학회지, 7: 130-139, 2018a.

김민주, 양병화, 김성문. 잡초의 향기가 소비자 행동에 미치는 영향. 한국잡초학회한국잔디학회지, $7: 98-110,2018 \mathrm{~b}$. 김선미, 김경란, 김란, 김봉인, 김선옥, 김해남, 모정희, 윤미숙, 장문정, 하명희. 아로마테라피 이론 및 실제. 정문각, 서 울, pp3-69. 2013.

남경돈, 민병찬, 정순철, 이동형, 민병운, 김유나, 김철중, 김준수. 향자극에 대한 뇌파의 시계열 분석. 한국감성과학회지, 2000: 44-47, 2000. 
민병찬. 인체에 미치는 향의 심리/생리적 효과. 한국정밀공학회지, 18: 46-53, 2001.

민병찬, 정순철, 김상균, 오지영, 김혜주, 김수진, 김유나, 신정상, 민병운, 김철중, 박세진. 뇌파와 자율신경계 반응을 이

용한 향(香)의 영향 평가. 한국감성과학회지, $2: 1-10,1999$.

이은정, 김유수, 김미선, 김은정, 김지수, 류현정, 문정숙, 박명자, 배근아, 차희연. 아로마테라피 완벽 가이드 $3 \mathrm{rd}$ 에디션.

대한아로마학회, 서울, pp221-223, 2019. (Battaglia S. 2018).

박미경, 정희윤, 이경화, 최정인, 이배환, 손진훈. 연령별 향 감성 구조 및 향 감성에 따른 자율신경계 반응. 한국감성과학 회지, 4: 39-45, 2001.

배익렬, 허명행. 에센셜오일 흡입이 스트레스와 교감신경 활성도에 미치는 효과. 대한스트레스학회지, 24: 75-83, 2016. 백은주, 이윤영, 이배환, 문창현, 이수환, 한희철. 뇌파와 자율신경계 반응에 나타난 오렌지향과 valeric acid에 의한 후각

감성. 한국감성과학회지, 1: 105-111, 1998.

신동준, 김주연. $\mathrm{EEG}$ 실험에 의한 발도르프학교 교실색채 반응 특성에 관한 연구. 한국디자인학회지, $31: 161-174$, 2018.

심은경, 김광옥, 이순례, 장윤선. 아로마 오일을 이용한 발 반사요법이 헤어 미용사의 스트레스 완화에 미치는 영향. 아시 안뷰티화장품학술지, 14: 369-378, 2016.

오윤경. 향 평가에 의한 아로마 에센셜오일의 유형화와 감성분석. 한국미용학회지, $18: 237-244,2012$.

오홍근, 최진영, 전겸구, 이준석, 박동기, 최성돈, 전태일. 3 종의 아로마 시너지브랜딩향의 항스트레스와 각성효과 및 3 종

향 사이의 효과에 비교 연구. 대한스트레스학회지, 8: 9-24, 2000.

용희정, 장현희, 이성내, 김수연, 김영삼. 중년여성을 대상으로 한 아로마테라피 실험연구 동향분석. 아시안뷰티화장품학 술지, 15: 113-121, 2017.

이경진, 이송이, 박소영, 장소라, 강창기. 구강 호흡이 뇌기능에 미치는 영향에 관한 EEG 연구. 한국감성과학회지, 19:

119-126, 2016.

이하나, 강승자. 아로마요법이 임상실습 중인 간호대학생의 스트레스, 피로및 심박변이도에 미치는 효과. 학습자중심교과 교육학회지, 19: 1415-1432, 2019.

이현주, 신동일, 신동규. 기계학습 알고리즘에 기반한 뇌파 데이터의 감정분류 및 정확도 향상에 관한 연구. 인터넷정보학

회논문지, 20: 27-36, 2019.

임미혜. 마조람(Origanum marjorana) 향유 흡입이 뇌파에 미치는 영향. 한국미용학회지, 17: 953-961, 2011.

장순복, 추상희, 김영인, 윤선희. 아로마 흡입이 밤번근무 간호사의 수면과 피로에 미치는 효과.성인간호학회지, 20 :

941-949, 2008.

정수현, 임미혜. 로만 카모마일의 흡입이 중년여성의 자율신경계에 미치는 영향. 한국미용학회지, 22: 636-643, 2016.

정은영, 송지현. 아로마 흡입이 중환자실 간호사의 스트레스, 우울 및 피로에 미치는 영향. 아시안뷰티화장품학술지, 16:

321-331, 2018.

최승완. 에센셜 아로마테라피. 의학서원, 서울, pp3-181, 2014.

한미라, 최정숙. Bergamot과 Grapefruit essential oil관리 시 자연광노출에 따른 표피층의 변화 연구. 한국미용학회지,

10: 202-208, 2004.

허선희, 유성모, 심준영. 추출부위에 따른 두 종류의 에센셜오일이 자율신경계에 미치는 영향. 한국미용학회지, $23: 461-$

474, 2017. 


\section{中文摘要}

\section{甜橙、薰衣草和阿米香精油对脑电活动的影响}

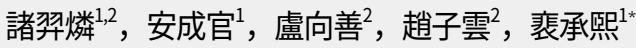

建国大学化妆品学科, 首尔, 韩国

${ }^{2}$ (株) Nowcos 研究中心, 首尔, 韩国

目的: 本研究评估了含有甜橙、薰衣草和杏仁的混合精油(mixed essential oils containing sweet orange, lavender, and amyris, MEO)对人类脑电图(EEG)活动的影响。方法: 通过检查20名年龄在20至 30岁之间的 成年人在吸入混合精油之前和期间的脑电波序列来记录EEG的活动。结果: MEO 显示出以额叶为中心的活动, 额叶负责对抗外部刺激的高阶功能，该结果表明油起到了智力作用。其他实验表明，大脑通过绝对慢阿尔法 (ASA)和相对慢阿尔法 (RSA)的减少、绝对贝塔 (AB) 和绝对高贝塔 (AHB) 的减少以及光谱边缘频率分别增加 $50 \%$ 的 alpha (ASEF)。此外, 吸入气味时, absolute slow alpha (ASA), relative slow alpha(RSA), absolute beta(AB), absolute high beta (AHB)减少, spectral edge frequency $50 \%$ of alpha (ASEF)指数增加, 通 过这个发现大脑变得轻松愉快。通过降低 absolute alpha(AA), absolute theta (AT)并增加 spectral edge frequency 50\% (SEF50), 可以确认大脑唤醒状态。通过 absolute fast alpha (AFA), relative fast alpha(RFA), relative mid beta(RMB), ratio of mid beta to theta (RMT), ratio of SMR to theta (RSMT), relative gamma (RG), spectral edge requency 90\% (SEF90)的增加, 可以确认大脑沉浸状态。结论: 综上所述, 这些结果表明 吸入 MEO 会影响大脑处于良好状态并提高其注意力集中能力。

关键词: 香气, 香薰, 脑电波, 脑电图, 精油 